\title{
Article \\ Prospective Evaluation of Side-Effects Following the First Dose of Oxford/AstraZeneca COVID-19 Vaccine among Healthcare Workers in Saudi Arabia
}

\author{
Majid A. Darraj ${ }^{1, *}$ and Hesham M. Al-Mekhlafi ${ }^{2,3, *}$ \\ Department of Internal Medicine, Faculty of Medicine, Jazan University, Jazan 45142, Saudi Arabia \\ Medical Research Centre, Jazan University, Jazan 45142, Saudi Arabia \\ Department of Parasitology, Faculty of Medicine and Health Sciences, Sana'a University, Sana'a 1247, Yemen \\ * Correspondence: mdarraj@jazanu.edu.sa (M.A.D.); halmekhlafi@jazanu.edu.sa (H.M.A.-M.)
}

check for updates

Citation: Darraj, M.A.; Al-Mekhlafi, H.M. Prospective Evaluation of Side-Effects Following the First Dose of Oxford/AstraZeneca COVID-19 Vaccine among Healthcare Workers in Saudi Arabia. Vaccines 2022, 10, 223. https://doi.org/10.3390/ vaccines 10020223

Academic Editors: P Veeranna Ravindra, Prashant Chikkahonnaiah and Federico Pratesi

Received: 6 December 2021

Accepted: 27 January 2022

Published: 30 January 2022

Publisher's Note: MDPI stays neutral with regard to jurisdictional claims in published maps and institutional affiliations.

Copyright: (c) 2022 by the authors. Licensee MDPI, Basel, Switzerland. This article is an open access article distributed under the terms and conditions of the Creative Commons Attribution (CC BY) license (https:// creativecommons.org/licenses/by/ $4.0 /)$.

\begin{abstract}
Background: Several different types of vaccines have been developed for the prevention of coronavirus disease (COVID-19). Despite several local and systemic side-effects to COVID-19 vaccination reported, the vaccines are still considered the best intervention to tackle the spread of the virus and reduce the severity of COVID-19 infection. However, the reported side-effects continue to have a crucial role in public confidence in the vaccine and its acceptance. This study aimed to investigate the short-term side-effects reported by the healthcare workers (HCWs) in Saudi Arabia after receiving the first dose of the Oxford/AstraZeneca (ChAdOx1 nCoV-19) COVID-19 vaccine. Methods: A prospective cohort study was conducted among HCWs in the Jazan region of southwestern Saudi Arabia. Healthcare workers who had received the first dose of the COVID-19 vaccine and agreed to participate in the study were followed up for 3 weeks post vaccination through a weekly online survey. Information was collected on local and/or systemic side-effects reported following vaccine administration. Participants' general and demographic information was also collected. Results: A total of 57.2\% (250/437) of the HCWs who participated in this study reported at least one side-effect. Injection site pain and redness (80.0\%), fever $(73.2 \%)$, whole-body pain/fatigue $(56.4 \%)$, and headache $(48.8 \%)$ were the most commonly reported side-effects. Moreover, $12.4 \%$ of the participants who reported side-effects needed to see a physician, and only one female participant was admitted to the hospital. Multivariate regression analyses revealed that nationality (Saudi, adjusted odds ratio $(\mathrm{aOR})=3.65 ; 95 \% \mathrm{CI}=2.40,5.55)$ and residency (Jazan governorate, $\mathrm{aOR}=0.38$; $95 \% \mathrm{CI}=0.15,0.95)$ were the significant factors associated with reporting COVID-19 post-vaccination side-effects, while the number of reported side-effects was found to be significantly influenced by occupation (medical, aOR $=0.42 ; 95 \% \mathrm{CI}=0.26,0.66 ; p<0.001$ ) and gender (female, aOR $=0.61$; $95 \% \mathrm{CI}=0.38,0.97 ; p=0.038)$. Conclusions: Findings of the present study support the safety of the Oxford/AstraZeneca COVID-19 vaccine among HCWs in Saudi Arabia. All the reported side-effects were mild-to-moderate side-effects. The findings may help convince vaccine-hesitant individuals and skeptics to accept the COVID-19 vaccine.
\end{abstract}

Keywords: COVID-19; vaccine; side-effects; healthcare workers; Saudi Arabia

\section{Introduction}

The novel coronavirus (COVID-19), caused by severe acute respiratory syndrome coronavirus 2 (SARS-CoV-2), was declared a global pandemic on 11 March 2020 [1]. Since then, most countries have taken precautions to stop COVID-19 from spreading in the hope of quickly developing safe and effective vaccines [2]. Extensive research has been condensed to bring a COVID-19 vaccine to market by late 2020 or early 2021, along with safety testing to prevent any serious side-effects [3]. Globally, Pfizer/BioNTech (BNT162b2) was the first vaccine authorized by the United States (US) Food and Drug Administration 
(FDA) in December 2020 [4], followed by Oxford / AstraZeneca being approved in January 2021 by the European Commission.

Many COVID-19 vaccine candidates have been developed by major global biopharmaceutical companies. The Pfizer/BioNTech Comirnaty vaccine was the first vaccine for COVID-19 listed on WHO's Emergency Use Listing (EUL) on 31 December 2020. The AstraZeneca AZD1222 and SII Covishield vaccines developed by AstraZeneca/Oxford were given EUL on 16 February 2021. Moreover, the Johnson and Johnson's Janssen Ad26.COV 2.S vaccine was listed for EUL on 12 March 2021, and the Moderna COVID-19 vaccine (mRNA 1273) was listed on 30 April 2021. The Sinopharm COVID-19 vaccine produced by Beijing Bio-Institute of Biological Products Co Ltd., a China National Biotec Group (CNBG) subsidiary, was listed for EUL on 7 May 2021. The Sinovac/CoronaVac was listed for EUL on 1 June 2021 [5].

These vaccines were developed using different technologies. The Pfizer/BioNTech BNT162b2 and Moderna mRNA 1273 COVID-19 vaccines are messenger RNA (mRNA)based vaccines, which encode SARS-CoV-2 prefusion-stabilized full-length spike protein [6], with efficacy rates of $95 \%$ and $94.1 \%$, respectively [7]. Likewise, the vaccines developed by Oxford/AstraZeneca and Johnson and Johnson are considered viral vector-based vaccines [8]. The Oxford/AstraZeneca vaccine consists of a replication-deficient chimpanzee adenoviral vector ChAdOx1 containing the SARS-CoV-2 structural surface glycoprotein antigen (spike protein; $\mathrm{nCoV-19}$ ) gene, with an efficacy rate of 70\% [9]. Both the Chinese vaccines (Sinopharm and Sinovac/CoronaVac) are inactivated vaccines, which use killed SARS-CoV-2 virus [8]. Overall, these vaccine types all met the necessary criteria for safety and efficacy as evaluated by the WHO [10].

However, these vaccines were linked to various adverse events, as concluded in clinical trials [11]. Moreover, several reports from different countries revealed that adverse events reported following the first and/or the second dose of COVID-19 vaccines included local side-effects, such as local pain at the site of injection, swelling, tenderness, redness, warmth, itch, and swollen armpit glands. They also included nonspecific systemic sideeffects, such as headache, fever, fatigue, night sweats, chills and shivering, joint pain, muscle pain, nausea, vomiting, and decreased appetite [6,12-17]. Despite being very rare, a few clinical trials reported serious and life-threatening adverse events, such as vaccineinduced immune thrombocytopenia and thrombosis (VIIT), following the first dose of certain vaccines, mainly with viral vector vaccines $[18,19]$. Nonetheless, a recent interim analysis of surveillance data from over six million individuals found no association between VIIT and mRNA vaccines [20]. More information on adverse events is required at the global level to understand better the factors associated with different vaccine-specific adverse events, which could help guide further investigations and proper clinical management of the cases.

In Saudi Arabia, COVID-19 cases reached 547,845 confirmed cases by mid-October 2021 and 8758 total deaths [21]. The country started its COVID-19 vaccination program in December 2020 to stop the spread of COVID-19 [22]. Three vaccines are currently available in Saudi Arabia: Pfizer/BioNTech, Oxford/AstraZeneca, and Moderna COVID19 vaccines [23]. About 45 million COVID-19 vaccine doses have been administered so far, with nearly $60 \%$ of the country's total population receiving two doses of the vaccines [24]. While there are data on COVID-19 vaccine views expressed by the general population and their acceptability of vaccination [25], data on the attitude of healthcare workers (HCWs) toward the Oxford/AstraZeneca and Pfizer/BioNTech COVID-19 vaccines are limited [26]. However, little is known about the factors associated with COVID-19 vaccine refusal or hesitancy. Within this context, the present study aimed to identify short-term side-effects among HCWs in Saudi Arabia after receiving their first dose of the Oxford/AstraZeneca COVID-19 vaccine (ChAdOx1 nCoV-19). 


\section{Materials and Methods}

\subsection{Study Settings}

A prospective cohort study was conducted among HCWs in the Jazan region of Saudi Arabia from May through June 2021. All vaccinated HCWs who had received their first dose of vaccination with the Oxford/AstraZeneca vaccine were followed up for 3 weeks post vaccination through a weekly online survey where the participants were asked to self-report any symptoms they developed. Those who had mild-to-moderate symptoms notified their clinic at the respective hospital via WhatsApp, SMS, or in person to set an appointment to be seen by a physician. A thorough assessment was performed and documented in their medical record for further follow-up by an infection control team for the specified 3 week period.

The target populations of the present study were all clinical (including physicians, nurses, medical technicians, and pharmacists) and nonclinical HCWs (including administrative and medical record professionals, workers, security, and nonmedical technicians) who were actively serving at participating private and public hospitals in Jazan region during the time of the study. The eligible participants were to have not received their first dose of COVID-19 vaccine before accepting the invitation to participate and filling in the questionnaire. Healthcare workers who were vaccinated before the study period were excluded. At the participating hospitals, an invitation message and information about the study objective and procedures were sent to all HCWs via their WhatsApp and email groups. Those who were eligible and willing to participate were listed, and their contact information was obtained. The participants were instructed to contact the survey team after they made appointments to get vaccinated.

Ethical approval was granted by the research ethics committee of Jazan University. A signed written informed consent was obtained from all participants prior to completing the survey. This procedure was approved by the research ethics committee. Participation in this survey was not compensated financially or by any other incentives.

\subsection{Sample Size}

The minimum sample size required for this survey was calculated according to Lwanga and Lemeshow [27]. As previous data on COVID-19 post-vaccination side-effects in the HCWs in Saudi Arabia were unavailable, a 0.50 assumed proportion was considered [28], with a $5 \%$ margin of error and $95 \%$ confidence level. Accordingly, 384 was yielded as the minimum number of participants required for this study. Considering a drop-out rate of $10 \%$, the final sample size was determined as 422 . During the study period, 485 eligible HCWs agreed to participate in the survey.

\subsection{Instrument and Data Collection}

A structured self-administered questionnaire of this survey was created after extensive review of the literature on adverse events of various COVID-19 vaccines [11,12,29]. The questionnaire was designed using Google Forms and was written in English and Arabic. The final versions were reviewed by four different experts in the field to check for face and content validity. The questions addressed the demographic characteristics of respondents (age, sex, job category, marital status, residence, and nationality), medical anamnesis data (history of chronic diseases, and medical treatment taken regularly) and COVID-19-related anamnesis data (history of previous diagnosis with COVID-19, the type of vaccine they received, and vaccination date). The questionnaire also included questions for reporting short-term local and systemic side-effects associated with the COVID-19 vaccine, time of onset post vaccination, the duration of symptoms, any medication that were taken, doctor's visits due to side-effects, and admission to the hospital. A thorough list of potential sideeffects, covering the most common symptoms reported by previous studies, was provided in the questionnaire. Moreover, an open section was also provided for reporting other unlisted symptoms which might have been experienced by the respondents. 
Participants who made appointments to get vaccinated and notified the survey team were followed up. The survey's link was sent to the participants through WhatsApp and/or e-mail. All participants were asked to respond to the survey; thus, the survey team members contacted those who did not respond weekly to identify and verify their responses. Only those who had received the first dose of Oxford/AstraZeneca vaccine and submitted the required questionnaires during the follow-up period were included in the final analysis. Out of 485 eligible participants, 41 (8.5\%) individuals were lost to follow-up during the study period. Therefore, $437 \mathrm{HCWs}$ were included in the final analysis.

\subsection{Data Analysis}

The data were exported from Google Forms (Mountain View, CA, USA) into Microsoft Excel (Redmond, WA, USA) and then exported into the Statistical Package for Social Sciences (SPSS) software version 21.0 (IBM, Inc., Armonk, NY, USA) for statistical analysis. For descriptive analysis, frequency and proportion were used to express the distribution of categorical variables while mean \pm standard deviation (SD) or median (interquartile range, IQR) were used to present quantitative variables. Before analysis, all quantitative variables were assessed for normality by Shapiro-Wilk test. Pearson's chi-square $\left(\chi^{2}\right)$ test or Fisher's exact test was used where appropriate to examine the association between reporting COVID-19 post-vaccination side-effects as the dependent variable and the independent variables (demographic and health background characteristics). Likewise, Mann-Whitney $\mathrm{U}$ and Kruskal-Wallis tests were used to assess the number of COVID-19 post-vaccination side-effects reported by the studied HCWs as an ordinal dependent variable according to the independent variables.

Moreover, multivariate logistic regression analysis was also used to investigate the factors associated with reporting of side-effects following the Oxford/AstraZeneca COVID19 vaccine, which was coded as a dummy dependent variable (yes $=1$ and no $=0$ ). In addition, multivariate ordinal logistic regression analysis was performed to assess the factors associated with the number of side-effects following the vaccine. All variables that showed associations with $p \leq 0.25$ in the univariate analyses were included in the multivariate logistic regression models [30]. Odds ratios (ORs) with a 95\% confidence interval (CI) were computed for different analyses. Statistical significance was defined as a $p$-value of less than 0.05. Where applicable, $p$-values were adjusted for multiple comparisons using the sequential Bonferroni correction (Bonferroni-Holm) method [31].

\section{Results}

\subsection{General Characteristics of Participants}

A total of 437 HCWs who were vaccinated with the first dose of the Oxford/AstraZeneca COVID-19 vaccine participated in the study. The mean \pm SD age of the participants was $33.6 \pm 7.3$ years; around two-thirds $(64.8 \%, n=283)$ of them were females, and $35.2 \%$ were males. Nurses were the dominant HCW group at 38.2\% $(n=167)$, followed by medical record professionals $(14.0 \%, n=61)$. The participants were from three governorates, namely, Jazan, Sabia, and Abu Arish, with the majority $(86.5 \%, 378 / 437)$ living in Jazan city. Participants who had been previously diagnosed with COVID-19 made up 12.6\% $(n=55)$, most of whom were females $(80.0 \%, 44 / 55)$. Only three participants $(0.7)$ had a history of chronic disease, both diabetes mellitus (DM) and hypertension. Table 1 shows the general demographic and health characteristics of the participants.

\subsection{Reported COVID-19 Vaccine Side-Effects}

The results of this study showed that $57.2 \%$ (250/437) of the HCWs who participated in this study reported at least one COVID-19 post-vaccination side-effect. The median (IQR) number of the reported side-effects was $3.0(2,4)$. Figure 1 shows that most HCWs reported four side-effects $(32.4 \%, 81 / 250)$, and $24 \%$ reported three side-effects, while six side-effects were reported by only $1.6 \%$. 
Table 1. General demographic and health characteristics of the participants $(n=437)$.

\begin{tabular}{|c|c|c|}
\hline \multicolumn{2}{|c|}{ Characteristics } & \multirow{2}{*}{$\begin{array}{c}n(\%) \\
169(38.7)\end{array}$} \\
\hline Age group (years) & $20-30$ & \\
\hline & $31-40$ & $202(46.2)$ \\
\hline & $41-50$ & $50(11.4)$ \\
\hline & $>50$ & $16(3.7)$ \\
\hline \multirow[t]{2}{*}{ Nationality } & Saudi & $187(42.8)$ \\
\hline & Non-Saudi & $250(57.2)$ \\
\hline \multirow[t]{2}{*}{ Gender } & Females & $283(64.8)$ \\
\hline & Males & $154(35.2)$ \\
\hline \multirow[t]{2}{*}{ Marital Status } & Married & $250(57.2)$ \\
\hline & Single & $187(42.8)$ \\
\hline \multirow[t]{3}{*}{ Residence } & Jazan & $378(86.5)$ \\
\hline & Sabia & $29(6.6)$ \\
\hline & Abu Arish & $30(6.9)$ \\
\hline \multirow[t]{9}{*}{ Occupation } & Nurse & $167(38.2)$ \\
\hline & Medical record professional & $61(14.0)$ \\
\hline & Administrative professional & $52(11.9)$ \\
\hline & $\begin{array}{c}\text { Workers (such as cleaning staff, } \\
\text { drivers, storekeepers) }\end{array}$ & $45(10.3)$ \\
\hline & Physician & $39(8.9)$ \\
\hline & Technician-medical & $37(8.5)$ \\
\hline & Security & $18(4.1)$ \\
\hline & Technician-nonmedical & $16(3.7)$ \\
\hline & Pharmacist & $2(0.5)$ \\
\hline \multirow[t]{2}{*}{$\begin{array}{l}\text { Diagnosed previously with } \\
\text { COVID-19 }\end{array}$} & Yes & $55(12.6)$ \\
\hline & No & $382(87.4)$ \\
\hline \multirow[t]{2}{*}{ History of chronic diseases } & DM and hypertension & $3(0.7)$ \\
\hline & No & $434(99.3)$ \\
\hline
\end{tabular}

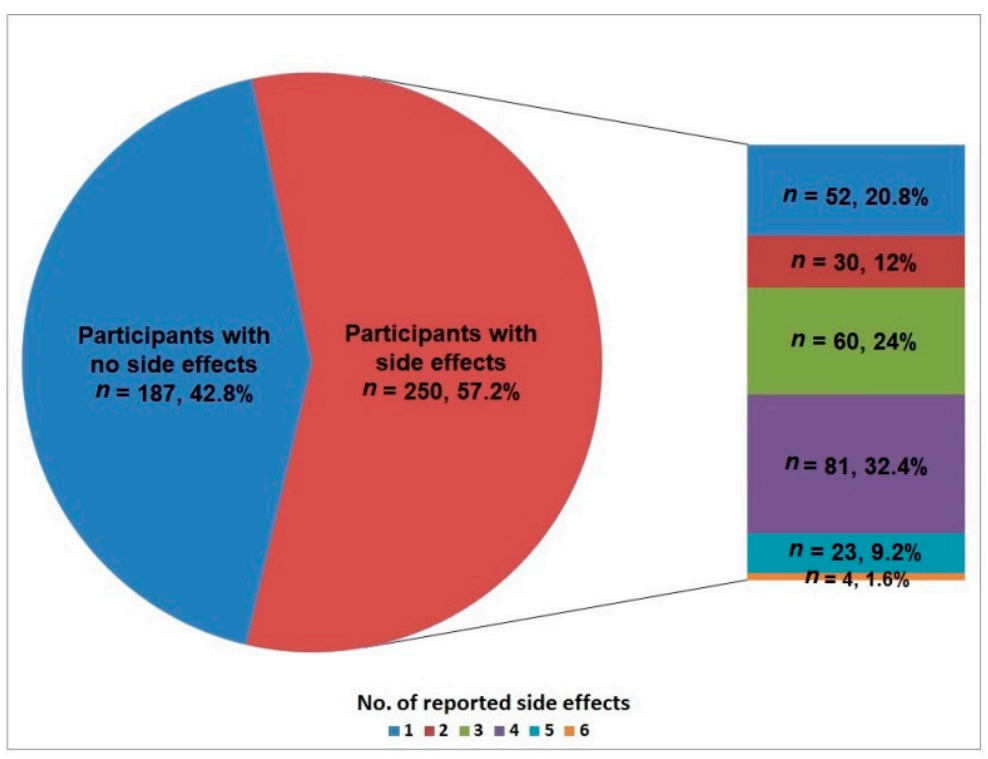

Figure 1. Total number of COVID-19 vaccine side-effects reported among the participants.

Among the $250 \mathrm{HCWs}$ who developed COVID-19 post-vaccination side-effects, Figure 2 shows that pain and redness at the site of injection $(80.0 \%)$ and fever $(73.2 \%)$ were the most frequently reported side-effects, followed by whole-body pain/fatigue (56.4\%) and headache $(48.8 \%)$. On the other hand, the least frequently reported side-effects were diarrhea at $1.2 \%$, shortness of breath, chest tightness, and vomiting at $0.8 \%$ each, and backache and skin rash at $0.4 \%$ each. 


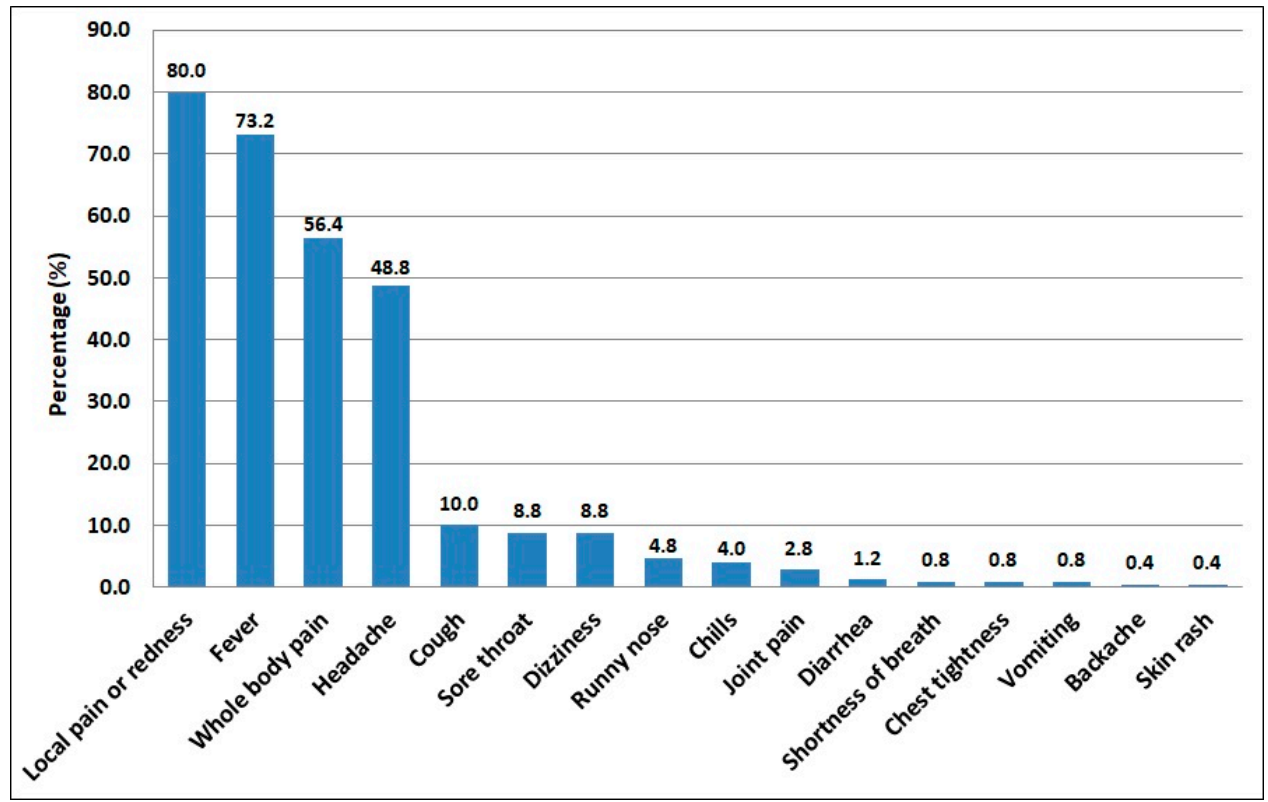

Figure 2. The frequency of COVID-19 vaccine side-effects reported among the participants $(n=250)$.

The distribution of different side-effects according to participants' gender was investigated among the HCWs who developed COVID-19 post-vaccination side-effects (Table 2). The results showed that female HCWs had a significantly higher frequency of side-effects of dizziness $\left(11.9 \%\right.$ vs. $\left.3.3 \% ; \chi^{2}=5.236 ; p=0.022\right)$ and chills $\left(6.2 \%\right.$ vs. $0.0 \% ; \chi^{2}=5.859$; $p=0.015)$ compared to male HCWs. On the other hand, the male HCWs had a significantly higher frequency of whole-body pain/fatigue $(67.8 \%, 61 / 90)$ compared to their female peers $(50.0 \%, 80 / 160)\left(\chi^{2}=7.403 ; p=0.007\right)$. Nonetheless, these significant differences were not retained when the Bonferroni-Holm adjustment was applied.

Table 2. Distribution of COVID-19 vaccine side-effects reported among the healthcare worker participants according to gender $(n=250)$.

\begin{tabular}{ccccc}
\hline Symptoms & $\begin{array}{c}\text { Male } \\
(\boldsymbol{n}=\mathbf{9 0})\end{array}$ & $\begin{array}{c}\text { Female } \\
(\boldsymbol{n}=\mathbf{1 6 0 )}\end{array}$ & $\begin{array}{c}\text { Total } \\
(\boldsymbol{n}=\mathbf{2 5 0 )}\end{array}$ & $\boldsymbol{\chi}^{\mathbf{2}(\boldsymbol{p})}$ \\
\hline $\begin{array}{c}\text { Pain or redness at the site } \\
\text { of injection }\end{array}$ & $72(80.0)$ & $128(80.0)$ & $200(80.0)$ & $0.001(0.999)$ \\
Fever & $65(72.2)$ & $118(73.8)$ & $183(73.2)$ & $0.069(0.793)$ \\
Whole body pain/fatigue & $61(67.8)$ & $80(50.0)$ & $141(56.4)$ & $7.403(0.007)$ \\
Headache & $51(56.7)$ & $71(44.4)$ & $122(48.8)$ & $3.483(0.062)$ \\
Cough & $13(14.4)$ & $12(7.5)$ & $25(10.0)$ & $3.086(0.079)$ \\
Sore throat & $12(13.3)$ & $10(6.2)$ & $22(8.8)$ & $3.601(0.058)$ \\
Dizziness & $3(3.3)$ & $19(11.9)$ & $22(8.8)$ & $5.236(0.022)$ \\
Runny nose & $6(6.7)$ & $6(3.8)$ & $12(4.8)$ & $1.072(0.301)^{\dagger}$ \\
Chills & $0(0.0)$ & $10(6.2)$ & $10(4.0)$ & $5.859(0.015)^{\dagger}$ \\
Joint pain & $2(2.2)$ & $5(3.1)$ & $7(2.8)$ & $0.172(0.587)^{\dagger}$ \\
Diarrhea & $1(1.1)$ & $2(1.2)$ & $3(1.2)$ & $0.009(0.735)^{\dagger}$ \\
Shortness of breath & $0(0.0)$ & $2(1.2)$ & $2(0.8)$ & $1.134(0.549)^{\dagger}$ \\
Chest tightness & $0(0.0)$ & $2(1.2)$ & $2(0.8)$ & $1.134(0.549)^{\dagger}$ \\
Vomiting & $0(0.0)$ & $2(1.2)$ & $2(0.8)$ & $1.134(0.549)^{\dagger}$ \\
Backache & $1(1.1)$ & $0(0.0)$ & $1(0.4)$ & $1.785(0.630)^{\dagger}$ \\
Skin rash & $0(0.0)$ & $1(0.6)$ & $1(0.4)$ & $0.565(0.710)^{\dagger}$ \\
\hline
\end{tabular}

$\chi^{2}$, chi-square test statistic. Significant difference between the two groups (unadjusted $\left.p<0.05\right) .{ }^{\dagger}$ Fisher's exact test statistic. 


\subsection{Associations of the Reported COVID-19 Vaccine Side-Effects}

Table 3 shows the associations of COVID-19 vaccine side-effects with participants' demographic and health background characteristics. The results showed comparable frequencies $(p=0.758)$ of HCWs who developed side-effects between female $(56.5 \%, 160 / 283)$ and male $(58.4 \%, 90 / 154)$ participants. Similarly, there was no significant difference in the frequency of side-effects according to participants' age $(p>0.05)$. The side-effects were significantly more frequent among Saudi HCWs $(73.8 \%, 138 / 187)$ compared to non-Saudis $(44.8 \%, 112 / 250)(p<0.001)$. Similarly, participants from the Jazan governorate had a significantly lower frequency of side-effects $(54.2 \%)$ than that reported for participants from the Abu Arish $(76.7 \%)$ and Sabia $(75.9 \%)$ governorates $(p<0.05)$. Interestingly, medical HCWs had a significantly lower frequency of side-effects than nonmedical HCWs $(51.0 \%$ vs. $65.1 \% ; p=0.003$ ). After Bonferroni-Holm correction, only two variables, nationality (Saudi) and occupation (medical), showed a significant association with the reporting of side-effects, while the significance of residence (Jazan) was lost (Table 2).

Table 3. Associations of the reported COVID-19 vaccine side-effects with the participants' background characteristics $(n=437)$.

\begin{tabular}{|c|c|c|c|c|}
\hline \multirow[t]{2}{*}{ Characteristic } & \multicolumn{2}{|c|}{ Reported Side-Effects } & \multirow[t]{2}{*}{ OR $(95 \% \mathrm{CI})$} & \multirow[t]{2}{*}{$p$} \\
\hline & Yes $(n=250)$ & No $(n=187)$ & & \\
\hline \multicolumn{5}{|l|}{ Age group (years) } \\
\hline $20-30(n=169)$ & $94(55.6)$ & $75(44.4)$ & 1 & \\
\hline $31-40(n=202)$ & $115(56.9)$ & $87(43.1)$ & $1.06(0.70,1.60)$ & 0.801 \\
\hline $41-50(n=50)$ & $29(58.0)$ & $21(42.0)$ & $1.10(0.58,2.09)$ & 0.766 \\
\hline$>50(n=16)$ & $12(75.0)$ & $4(25.0)$ & $2.39(0.74,7.72)$ & 0.134 \\
\hline \multicolumn{5}{|l|}{ Gender } \\
\hline Female $(n=283)$ & $160(56.5)$ & $123(43.5)$ & $0.93(0.62,1.38)$ & 0.701 \\
\hline \multicolumn{5}{|l|}{ Nationality } \\
\hline Saudi $(n=190)$ & $138(73.8)$ & $49(26.2)$ & $3.47(2.30,5.23)$ & $<0.001$ * \\
\hline \multicolumn{5}{|l|}{ Marital status } \\
\hline Married $(n=255)$ & 145 (57.2) & $105(42.0)$ & $1.08(0.74,1.58)$ & 0.699 \\
\hline $\begin{array}{l}\text { Single }(n=189) \\
\text { Residence }\end{array}$ & $105(56.1)$ & $82(43.9)$ & 1 & \\
\hline Jazan $(n=378)$ & $205(54.2)$ & $173(45.8)$ & $0.36(0.15,0.86)$ & 0.017 \\
\hline Sabia $(n=29)$ & $22(75.9)$ & $7(24.1)$ & $0.96(0.29,3.18)$ & 0.924 \\
\hline $\begin{array}{l}\text { Abu Arish }(n=30) \\
\text { Occupation }\end{array}$ & $23(76.7)$ & $7(23.3)$ & 1 & \\
\hline Medical $(n=245)$ & $125(51.0)$ & $120(49.0)$ & $0.56(0.38,0.82)$ & $0.003 *$ \\
\hline Nonmedical $(n=192)$ & $125(65.1)$ & $67(34.9)$ & 1 & \\
\hline \multicolumn{5}{|l|}{$\begin{array}{l}\text { Diagnosed previously } \\
\text { with COVID-19 }\end{array}$} \\
\hline Yes $(n=55)$ & $28(50.9)$ & $27(49.1)$ & $0.75(0.43,1.32)$ & 0.313 \\
\hline No $(n=382)$ & $222(58.1)$ & $160(41.9)$ & 1 & \\
\hline
\end{tabular}

(using the Bonferroni-Holm correction for multiple comparisons).

Differences in the number of COVID-19 post-vaccination side-effects reported by the studied HCWs according to independent variables were examined, and the results are shown in Table 4. A Mann-Whitney $U$ test showed that the number of side-effects was found to be significantly lower among the medical HCWs (median $=3 ; \mathrm{IQR}=1,4$ ) compared to the nonmedical HCWs (median $=4 ; \mathrm{IQR}=3,4)(\mathrm{U}=5874 ; p<0.001)$. On the other hand, the Kruskal-Wallis test results showed no significant difference in the number of side-effects according to age groups $(\mathrm{H}=4.079 ; p=0.245)$. Similarly, differences in the number of side-effects according to other variables were not significant $(p>0.05)$. 
Table 4. Number of side-effects reported by the participants following the first dose of COVID-19 vaccine according to the participants' background characteristics $(n=250)$.

\begin{tabular}{|c|c|c|c|}
\hline Characteristic & $\begin{array}{l}\text { No. of Reported } \\
\text { Side-Effects }\end{array}$ & Statistics & $p$ \\
\hline \multicolumn{4}{|c|}{ Median (IQR) } \\
\hline Age group (years) & & $\mathrm{H}=4.079$ & 0.245 \\
\hline $20-30(n=94)$ & $3(2,4)$ & & \\
\hline $31-40(n=115)$ & $3(2,4)$ & & \\
\hline $41-50(n=29)$ & $3(1,4)$ & & \\
\hline$>50(n=12)$ & $2(1,3)$ & & \\
\hline Gender & & $\mathrm{U}=6366$ & 0.117 \\
\hline Female $(n=90)$ & $3(2,4)$ & & \\
\hline Male $(n=160)$ & $3(2,4)$ & & \\
\hline Nationality & & $\mathrm{U}=7643$ & 0.878 \\
\hline Saudi $(n=138)$ & $2(3,4)$ & & \\
\hline Non-Saudi $(n=112)$ & $2(3,4)$ & & \\
\hline Marital status & & $\mathrm{U}=7475$ & 0.802 \\
\hline Married $(n=145)$ & $3(2,4)$ & & \\
\hline Single $(n=105)$ & $3(2,4)$ & & \\
\hline Residence & & $\mathrm{H}=2.125$ & 0.346 \\
\hline Jazan $(n=205)$ & $3(2,4)$ & & \\
\hline Sabia $(n=22)$ & $3(2,4)$ & & \\
\hline Abu Arish $(n=23)$ & $4(3,4)$ & & \\
\hline Occupation & & $\mathrm{U}=5874$ & $<0.001 *$ \\
\hline Medical $(n=125)$ & $3(1,4)$ & & \\
\hline Nonmedical $(n=125)$ & $4(3,4)$ & & \\
\hline $\begin{array}{l}\text { Diagnosed previously } \\
\text { with COVID-19 }\end{array}$ & & $\mathrm{U}=3062$ & 0.896 \\
\hline Yes $(n=28)$ & $3(3,4)$ & & \\
\hline No $(n=222)$ & $3(2,4)$ & & \\
\hline
\end{tabular}

$\overline{\mathrm{IQR}}$, interquartile range; U, Mann-Whitney U statistic; H, Kruskal-Wallis test statistic. Significant difference (unadjusted $p<0.05$ ). * Significant difference (using the Bonferroni-Holm correction for multiple comparisons).

\subsection{Multivariate Analyses of Factors Associated with the Reported COVID-19 Vaccine Side-Effects}

Table 5 presents the multivariate logistic regression analysis results for the factors associated with reporting side-effects following the first dose of the COVID-19 vaccine among the HCWs studied. All variables that showed associations with $p \leq 0.25$ in the univariate analysis presented in Table 3 were included. The Hosmer-Lemeshow test, used for the inferential goodness-of-fit test, showed that the model fit the data well $\left(\chi^{2}=4.909\right.$; $p=0.427)$. The results revealed that Saudi HCWs had a 3.65-fold increased risk of sideeffects compared to non-Saudi HCWs (adjusted odds ratio $(\mathrm{aOR})=3.65 ; 95 \% \mathrm{CI}=2.40,5.55$ ). Moreover, HCWs who lived in the Jazan governorate had lower odds of reporting COVID19 post-vaccination side-effects than those who lived in Abu Arish or Sabia $(\mathrm{aOR}=0.38$; $95 \% \mathrm{CI}=0.15,0.95)$. However, the significant association of occupation with side-effects was not retained in the multivariate analysis $(p=0.067)$.

Table 5. Multivariate analysis of factors associated with reporting of side-effects following COVID-19 vaccine among the participants $(n=437)$.

\begin{tabular}{cccc}
\hline Variable & aOR & $\mathbf{9 5 \% ~ C I ~}$ & $p$ \\
\hline Nationality (Saudi) & 3.65 & $2.40,5.55$ & $<0.001^{*}$ \\
Residence (Jazan) & 0.38 & $0.15,0.95$ & $0.038^{*}$ \\
Occupation (medical) & 0.67 & $0.43,1.02$ & 0.062
\end{tabular}

aOR, adjusted odds ratio; CI, confidence interval. ${ }^{*}$ Significant association of COVID-19 vaccine side-effects $(p<0.05)$. 
Furthermore, Table 6 shows the multivariate ordinal logistic regression analysis results for the factors influencing the number of COVID-19 vaccine side-effects reported by the studied HCWs. All variables that showed differences with $p \leq 0.25$ in the univariate analysis presented in Table 4 were included in the model. The model was of good fit and statistically significant $\left(\chi^{2}=19.672, p<0.001\right)$. The number of reported side-effects was found to be significantly influenced by the occupation and gender of HCWs. The results showed that, holding other variables unchanged, medical HCWs were less likely to report a higher number of side $=$ effects than their nonmedical peers $(\mathrm{aOR}=0.42 ; 95 \% \mathrm{CI}=0.26$, $0.66 ; p<0.001)$. Similarly, female participants were less likely to report a higher number of side $=$ effects than male participants $(\mathrm{aOR}=0.61 ; 95 \% \mathrm{CI}=0.38,0.97 ; p=0.038)$.

Table 6. Results of ordinal logistic regression for the factors associated with the number of reported side-effects following COVID-19 vaccine $(n=250)$.

\begin{tabular}{cccc}
\hline Variable & aOR & $\mathbf{9 5 \%}$ CI & $p$ \\
\hline Age (year) & 0.97 & $0.94,1.49$ & 0.093 \\
Gender (female) & 0.61 & $0.38,0.97$ & $0.038^{*}$ \\
Occupation (medical) & 0.42 & $0.26,0.66$ & $<0.001 *$ \\
\hline aOR adjusted odds ratio; CI, confidence interval * Significant association $(p<0.05)$ &
\end{tabular}

aOR, adjusted odds ratio; CI, confidence interval. * Significant association $(p<0.05)$.

\subsection{Duration and Management of the Reported COVID-19 Vaccine Side-Effects}

Among the HCWs who developed COVID-19 post-vaccination side-effects, 93.6\% $(234 / 250)$ of the reported side-effects occurred on the day of receiving the vaccine (Day 0$)$. In comparison, $5.6 \%$ and $0.8 \%$ of the reported side-effects occurred Day 1 and Day 2 post vaccination (the second and third post-vaccination days), respectively. The duration of reported side-effects was $2-3$ days for $53.2 \%(n=133)$ and $4-5$ days for $26.0 \%(n=65)$, while the side-effects lasted for more than 7 days for only $2.4 \%(n=6)$ of the subset of participants who reported side-effects. Moreover, $12.4 \%(n=31)$ of those who developed side-effects visited a doctor, and medication for post-vaccination side-effects was taken by $66.4 \%(n=166)$. Only one female participant $(0.4 \%)$ was admitted to the hospital (Table 7$)$.

Table 7. The onset, duration, and management of COVID-19 vaccine side-effects reported among the healthcare workers participated in the study $(n=250)$.

\begin{tabular}{ccccc}
\hline $\begin{array}{c}\text { Onset and Duration of } \\
\text { Side-Effects }\end{array}$ & $\begin{array}{c}\text { Male } \\
(\boldsymbol{n}=\mathbf{9 0 )}\end{array}$ & $\begin{array}{c}\text { Female } \\
(\boldsymbol{n}=\mathbf{1 6 0 )})\end{array}$ & $\begin{array}{c}\text { Total } \\
(\boldsymbol{n}=\mathbf{2 5 0})\end{array}$ & $\boldsymbol{\chi}^{\mathbf{2}(\boldsymbol{p})}$ \\
\hline Onset & & & $0.172(0.918)$ \\
Day 0 & $84(93.3)$ & $150(93.8)$ & $234(93.6)$ & \\
Day 1 & $5(5.6)$ & $9(5.6)$ & $14(5.6)$ & \\
Day 2 & $1(1.1)$ & $1(0.6)$ & $2(0.8)$ & $5.196(0.268)$ \\
One day & & & \\
2-3 days & $8(8.9)$ & $20(12.5)$ & $28(11.2)$ & \\
4-5 days & $46(51.1)$ & $87(54.4)$ & $133(53.2)$ & \\
6-7 days & $30(33.3)$ & $35(21.9)$ & $65(26.0)$ & \\
More than 7 days & $4(4.4)$ & $14(8.8)$ & $18(7.2)$ & \\
Muration of symptoms & $2(2.2)$ & $4(2.5)$ & $6(2.4)$ & $0.390(0.532)$ \\
Doctication taken for side-effects & $62(68.9)$ & $104(65.0)$ & $166(66.4)$ & $0.215(0.643)$ \\
Hospitalization due to side-effects & $10(11.1)$ & $21(13.1)$ & $31(12.4)$ & $0.565(0.641)$ \\
\hline
\end{tabular}

$\chi^{2}$, chi-square test statistic; ${ }^{+}$Fisher's exact test statistic.

\section{Discussion}

This study aimed to identify short-term side-effects following the Oxford/AstraZeneca COVID-19 vaccine among Saudi Arabian HCWs in Jazan region. It is essential to assure COVID-19 vaccine recipients at this critical stage of the vaccination campaigns by collecting evidence-based data concerning the vaccines' side-effects, particularly if they are transient 
or temporary. Such evidence can alleviate fears and encourage the completion of the two-dose vaccination series [32].

The present study showed that $57.2 \%(250 / 437)$ of the studied HCWs reported at least one side-effect following the administration of the first dose of the Oxford/AstraZeneca COVID-19 vaccine. This finding is lower than recent findings reported in the Jazan region among the general population aged between 18 and 70 years who received the same type of COVID-19 vaccine (66.2\%; 255/385) [33]. In a study conducted in Abha, Aseer Region, southwestern Saudi Arabia among 167 individuals aged 18 years and older who received the Oxford/AstraZeneca vaccine, 74.3\% reported side-effects [29]. In contrast, a study among 1592 individuals in Dhahran City, northeastern Saudi Arabia, reported a lower incidence (34.7\%) of side-effects following the Oxford/AstraZeneca vaccine [34]. Interestingly, Alghamdi et al. [35] found that adverse events and severity following the first dose of Oxford/AstraZeneca vaccine were more common in HCWs than non-HCW participants. Indeed, this difference could be attributed to psychosocial factors, as HCWs may be more sensitive to side-effects due to their health education than non-HCWs [36]. Moreover, due to their high occupational exposure to COVID-19, the likelihood of a previous asymptomatic COVID-19 infection among HCWs may lead to a stronger immune response compared to the general population [35]. Individuals who had been previously diagnosed with COVID-19 were more likely to report more side-effects after the first dose of the COVID-19 vaccine [15,37]. However, the present study found no considerable association between the development of side-effects and previous COVID-19 infection among the studied HCWs, and this agrees with previous studies in Saudi Arabia [33,38].

On the other hand, recent studies conducted in other countries reported higher frequencies of side-effects following the Oxford/AstraZeneca COVID-19 vaccine among different population groups. For instance, $97.8 \%$ of 197 Jordanian HCWs who received the first or the second dose of the Oxford/AstraZeneca COVID-19 vaccine reported some side-effects [39]. Likewise, another study from Poland showed that $96.5 \%$ of 705 participants reported at least one post-vaccination side-effect [40]. A slightly higher frequency of COVID-19 post-vaccination side-effects among HCWs was also reported in India $(65 \%, 3556 / 5396)$ [41]. These differences could be attributed to different factors, including the incidence of COVID-19, the time these studies were conducted in relation to the pandemic waves and stages, and other demographic and health characteristics of the studied populations.

The side-effects can be local, such as injection site pain or redness, which was the most frequent side-effect reported by the studied participants, or systemic, including all remaining side-effects. Of these, fever was the second most frequently reported sideeffect. The most common side-effects in the present study were injection site pain and redness, fever, fatigue, headaches, cough, sore throat, and dizziness. Less common sideeffects were diarrhea, shortness of breath, chest tightness, vomiting, backache, and skin rash. These findings are similar to those reported among the general population in Saudi Arabia $[33,38]$. In comparison with studies conducted elsewhere, the reported frequencies of these side-effects were slightly higher than those reported by similar studies among HCWs in Jordan [39] and India [41]. On the other hand, higher frequencies of these sideeffects were reported among the general population in Poland [40]. The present study found no severe complications linked to the first dose of the Oxford/AstraZeneca COVID19 vaccine, and this agrees with previous studies in Saudi Arabia [29,33]. Nonetheless, serious adverse events such as severe allergic reaction, cardiac arrest, cerebral venous sinus thrombosis, and pulmonary embolism associated with the COVID-19 vaccine have been reported worldwide, including in Saudi Arabia [42,43].

In the current study, $93.3 \%$ of the side-effects occurred on the day of vaccination, and $35.6 \%(89 / 250)$ of the symptoms lasted for more than 3 days, which is consistent with the findings reported in other similar studies $[33,39]$. In the current survey, about two-thirds $(66.4 \%, 166 / 250)$ of participants took medication (mainly analgesics) to relieve the side-effects. Taking analgesics to alleviate the side-effects associated with the COVID-19 
vaccine is common among both HCWs and non-HCW populations in Saudi Arabia [35]. Moreover, only $12.4 \%$ of the participants needed to see a physician due to side-effects from the vaccines, and only one female participant was admitted to the hospital. This strongly supports the safety of these vaccines.

Regarding associations of post-vaccination side-effects with some demographic and health variables, the present study found that nationality and residence of the participants were the significant factors associated with the reporting of side-effects. Saudi HCWs had 3.65 times the odds of reporting side-effects compared to non-Saudi HCWs. This might be attributed to the perception of vaccine safety or the psychological and immunological status of the participants. Previous studies found that non-Saudi participants were more inclined to accept the COVID-19 vaccine [22]. They were also more likely to increase their physical activity during COVID-19 quarantine and mobility restrictions than their Saudi counterparts [44]. Moreover, the present study found that HCWs who lived in the Jazan governorate had lower odds of reporting COVID-19 post-vaccination side-effects than those who lived in Abu Arish or Sabia. While the explanation for this association is unknown, it might be attributed to the fact that the majority $(86.5 \%)$ of the participants involved in this study were from the Jazan governorate.

Furthermore, the present study showed that medical HCWs (including nurses, physicians, medical technicians, and pharmacists) had lower odds of reporting post-vaccination side-effects than nonmedical HCWs (including administrative and medical record professionals, workers, security, and nonmedical technicians). However, this association was not retained when logistic regression analysis was applied. Interestingly, the present study demonstrated that medical HCWs were likely to report a higher number of side-effects than the nonmedical HCWs. These findings could be attributed to the higher level of medical knowledge about vaccine safety among the medical HCWs that would enhance their ability to identify and differentiate the symptoms.

The present study found comparable frequencies of reporting side-effects for male and female participants, in agreement with previous studies [39]. However, this finding contradicts that reported by Alhazmi et al. [33], who found a higher incidence of sideeffects among female participants than male participants. In contrast, a study among 330 individuals in southwestern Saudi Arabia reported a higher incidence of side-effects in males than females following Pfizer/BioNTech and Oxford/AstraZeneca vaccination [29]. Interestingly, when ordinal logistic regression analysis was applied in the present study, the female gender was identified as a significant factor associated with reporting a lower number of side-effects compared to the male gender. This finding is consistent with recent studies conducted among 1592 individuals in Dhahran City, northeastern Saudi Arabia [34]. However, the finding is inconsistent with that of other reports from Saudi Arabia [38,45] and elsewhere $[14,15,46]$, which suggested that female gender was a significant factor for the development of more severe and a higher number of COVID-19 post-vaccination adverse events. However, findings on the gender-related differences of COVID-19 vaccine side-effects are still inconclusive.

Indeed, previous studies have suggested a gender-based difference in reporting adverse events following various viral and bacterial vaccines [47,48]. It is found that females typically mount stronger inflammatory, antibody, and cell-mediated immune responses to vaccines when compared with males, and this might explain the sex-based differences in reactions and immunogenicity toward vaccinations [46]. Furthermore, behavioral, genetic, and hormonal factors might also underlie the gender-based variation in adverse events following vaccination $[46,48,49]$. In addition, a few studies have reported significant associations between the development of post-vaccination side-effects and age. Studies among HCWs in Jordan [39], Germany [14], and Slovakia [50] found that young adult participants had a significantly higher incidence of COVID-19 vaccine side-effects compared to their older age counterparts, and this has been attributed to the fact that immune responses gradually weaken with age. Nevertheless, in agreement with some studies conducted in Saudi Arabia [33] and elsewhere [51], the present study did not find such an association. 
Some limitations of the current study should be acknowledged to be considered when interpreting the findings. First, this study used an online self-administered questionnaire instead of face-to-face interviews, which may result in reporting bias. However, the HCWs participants are expected to have adequate knowledge about the vital importance of the COVID-19 vaccine and its associated adverse events. Second, this study reported only short-term side-effects, while the vaccines' intermediate and long-term side-effects were not studied. Similarly, only side-effects following the first dose of the vaccine were studied, while the side-effects following the second dose of the vaccine were not evaluated. Furthermore, the study was also predominated by Oxford/AstraZeneca recipients with only a few Pfizer/BioNTech recipients. This could have been due to the shortage of the BioNTech vaccine during the study duration and the relatively small sample size; thus, further multicenter studies with larger sample sizes are warranted to ascertain the safety profile of different COVID-19 vaccines approved for use in the country.

\section{Conclusions}

The present study provides important information on the side-effects following COVID-19 vaccination among healthcare workers in Saudi Arabia. The findings showed that most participants reported pain at the injection site, fever, fatigue, and headache. Moreover, nationality (Saudi) and residence (Jazan) were identified as the significant factors associated with the reporting of side-effects following the first dose of the Oxford/AstraZeneca COVID-19 vaccine, while gender (female) and occupation (medical) were significantly associated with reporting a lower number of side-effects. The findings revealed that all the COVID-19 post-vaccination side-effects were mild to moderate, and only a small portion of vaccine recipients $(12.4 \%)$ needed to see a physician, while only one participant $(0.4 \%)$ admitted to the hospital due to those side-effects. Therefore, the current study's findings support the vaccine's safety and provide important baseline data to increase healthcare workers' and the general community's awareness of the expected side-effects following COVID-19 vaccines. This might help convince the vaccine-hesitant individuals and skeptics to accept the COVID-19 vaccine.

Author Contributions: Conceptualized the study, and conducted the survey and data collection, M.A.D.; data analysis and writing the manuscript, M.A.D. and H.M.A.-M. All authors have read and agreed to the published version of the manuscript.

Funding: This research received no external funding.

Institutional Review Board Statement: The current study was conducted according to the guidelines proposed by the Declaration of Helsinki, and the protocol was ethically approved by the research ethics committee of Jazan University (Ref. No. REC42/1/154). Before the commencement of data collection, written informed consent was obtained from each participant. This procedure was approved by the research ethics committee.

Informed Consent Statement: Written informed consent was obtained from all participants involved in the study.

Data Availability Statement: The data that support the findings of this study are available from the authors upon reasonable request.

Acknowledgments: The authors would like to thank all participants for their fruitful cooperation and support.

Conflicts of Interest: The authors declare no conflict of interest.

\section{References}

1. Cucinotta, D.; Vanelli, M. WHO declares COVID-19 a pandemic. Acta Biomed. Atenei Parm. 2020, 91, 157-160. [CrossRef]

2. Algaissi, A.A.; Alharbi, N.K.; Hassanain, M.; Hashem, A.M. Preparedness and response to COVID-19 in Saudi Arabia: Building on MERS experience. J. Infect. Public Health 2020, 13, 834-838. [CrossRef] [PubMed]

3. Raja, A.A.T.A.; Alshamsan, A.; Al-jedai, A. Current COVID-19 vaccine candidates: Implications in the Saudi population. Saudi Pharm. J. 2020, 28, 1743-1748. [CrossRef] [PubMed] 
4. FDA US. Comirnaty and Pfizer-BioNTech COVID-19 Vaccine. Maryland: The United States Food and Drug Administration. 2021. Available online: https://www.fda.gov/emergency-preparedness-and-response/coronavirus-disease-2019-COVID-19 / comirnaty-and-pfizer-biontech-COVID-19-vaccine (accessed on 15 October 2021).

5. WHO. Coronavirus Disease (COVID-19): COVID-19 Vaccines; World Health Organization: Geneva, Switzerland, 2021. Available online: https:/ / www.who.int/emergencies/diseases/novel-coronavirus-2019/COVID-19-vaccines (accessed on 10 November 2021).

6. El-Shitany, N.A.; Harakeh, S.; Badr-Eldin, S.M.; Bagher, A.M.; Eid, B.; Almukadi, H.; Alghamdi, B.S.; Alahmadi, A.A.; Hassan, N.A.; Sindi, N.; et al. Minor to moderate side effects of Pfizer-Biontech COVID-19 vaccine among Saudi residents: A retrospective cross-sectional study. Int. J. Gen. Med. 2021, 14, 1389-1401. [CrossRef]

7. Cavaleri, M.; Enzmann, H.; Straus, S.; Cooke, E. The European Medicines Agency's EU conditional marketing authorisations for COVID-19 vaccines. Lancet 2021, 397, 355-357. [CrossRef]

8. Mallapaty, S. China's COVID vaccines have been crucial-now immunity is waning. Nature 2021, 598, 398-399. [CrossRef]

9. Lopez Bernal, J.; Andrews, N.; Gower, C.; Robertson, C.; Stowe, J.; Tessier, E.; Simmons, R.; Cottrell, S.; Roberts, R.; O'Doherty, M.; et al. Effectiveness of the Pfizer-BioNTech and Oxford-AstraZeneca vaccines on COVID-19 related symptoms, hospital admissions, and mortality in older adults in England: Test negative case-control study. BMJ 2021, 373, n1088. [CrossRef]

10. WHO. COVID-19 Advice for the Public: Getting Vaccinated; World Health Organization: Geneva, Switzerland, 2021. Available online: https:/ / www.who.int/emergencies/diseases/novel-coronavirus-2019/COVID-19-vaccines/advice (accessed on 2 November 2021).

11. Polack, F.P.; Thomas, S.J.; Kitchin, N.; Absalon, J.; Gurtman, A.; Lockhart, S.; Perez, J.L.; Pérez Marc, G.; Moreira, E.D.; Zerbini, C.; et al. Safety and Efficacy of the BNT162b2 mRNA COVID-19 Vaccine. N. Engl. J. Med. 2020, 383, $2603-2615$. [CrossRef]

12. Kaur, R.J.; Dutta, S.; Bhardwaj, P.; Charan, J.; Dhingra, S.; Mitra, P.; Singh, K.; Yadav, D.; Sharma, P.; Misra, S. Adverse events reported from COVID-19 vaccine trials: A systematic review. Indian J. Clin. Biochem. 2021, 36, 427-439. [CrossRef]

13. Abu-Halaweh, S.; Alqassieh, R.; Suleiman, A.; Al-Sabbagh, M.Q.; AbuHalaweh, M.; AlKhader, D.; Abu-Nejem, R.; Nabulsi, R.; Al-Tamimi, M.; Alwreikat, M.; et al. Qualitative assessment of early adverse effects of Pfizer-BioNTech and Sinopharm COVID-19 vaccines by telephone interviews. Vaccines 2021, 9, 950. [CrossRef]

14. Klugar, M.; Riad, A.; Mekhemar, M.; Conrad, J.; Buchbender, M.; Howaldt, H.P.; Attia, S. Side effects of mRNA-based and viral vector-based COVID-19 vaccines among German healthcare workers. Biology 2021, 10, 752. [CrossRef] [PubMed]

15. Menni, C.; Klaser, K.; May, A.; Polidori, L.; Capdevila, J.; Louca, P.; Sudre, C.H.; Nguyen, L.H.; Drew, D.A.; Merino, J.; et al. Vaccine side-effects and SARS-CoV-2 infection after vaccination in users of the COVID Symptom Study app in the UK: A prospective observational study. Lancet Infect. Dis. 2021, 21, 939-949. [CrossRef]

16. Saeed, B.Q.; Al-Shahrabi, R.; Alhaj, S.S.; Alkokhardi, Z.M.; Adrees, A.O. Side effects and perceptions following Sinopharm COVID-19 vaccination. Int. J. Infect. Dis. 2021, 111, 219-226. [CrossRef]

17. Thomas, S.J.; Moreira, E.D.; Kitchin, N., Jr.; Absalon, J.; Gurtman, A.; Lockhart, S.; Perez, J.L.; Pérez Marc, G.; Polack, F.P.; Zerbini, C.; et al. Safety and efficacy of the BNT162b2 mRNA COVID-19 vaccine through 6 months. N. Engl. J. Med. 2021, 385, 1761-1773. [CrossRef] [PubMed]

18. See, I.; Su, J.R.; Lale, A.; Woo, E.J.; Guh, A.Y.; Shimabukuro, T.T.; Streiff, M.B.; Rao, A.K.; Wheeler, A.P.; Beavers, S.F.; et al. US case reports of cerebral venous sinus thrombosis with thrombocytopenia after Ad26.COV2.S vaccination, March 2 to April 21, 2021. JAMA 2021, 325, 2448-2456. [CrossRef] [PubMed]

19. Tobaiqy, M.; MacLure, K.; Elkout, H.; Stewart, D. Thrombotic adverse events reported for Moderna, Pfizer and OxfordAstraZeneca COVID-19 vaccines: Comparison of occurrence and clinical outcomes in the eudravigilance database. Vaccines 2021, 9, 1326. [CrossRef]

20. Klein, N.P.; Lewis, N.; Goddard, K.; Fireman, B.; Zerbo, O.; Hanson, K.E.; Donahue, J.G.; Kharbanda, E.O.; Naleway, A.; Nelson, J.C.; et al. Surveillance for adverse events after COVID-19 mRNA vaccination. JAMA 2021, 326, 1390-1399. [CrossRef]

21. MOH. MOH News. Riyadh: Ministry of Health. 2021. Available online: https://www.moh.gov.sa/en/Ministry/MediaCenter/ News/Pages/News-2021-10-15-001.aspx (accessed on 20 October 2021).

22. Elharake, J.A.; Galal, B.; Alqahtani, S.A.; Kattan, R.F.; Barry, M.A.; Temsah, M.H.; Malik, A.A.; McFadden, S.M.; Yildirim, I.; Khoshnood, K.; et al. COVID-19 vaccine acceptance among health care workers in the Kingdom of Saudi Arabia. Int. J. Infect. Dis. 2021, 109, 286-293. [CrossRef]

23. MOH. COVID-19 \& Vaccine FAQs. Riyadh: Ministry of Health. 2021. Available online: https://www.moh.gov.sa/en/Ministry/ HotTopics/Pages/COVID-19-Vaccine.aspx (accessed on 5 January 2022).

24. Our World in Data. Saudi Arabia: Coronavirus Pandemic Country Profile. 2021. Available online: https://ourworldindata.org/ coronavirus / country/saudi-arabia (accessed on 25 October 2021).

25. Al-Mohaithef, M.; Padhi, B.K. Determinants of COVID-19 vaccine acceptance in Saudi Arabia: A web-based national survey. J. Multidiscip. Healthc. 2020, 13, 1657-1663. [CrossRef]

26. Barry, M.; Temsah, M.H.; Aljamaan, F.; Saddik, B.; Al-Eyadhy, A.; Alenezi, S.; Alamro, N.; Alhuzaimi, A.N.; Alhaboob, A.; Alhasan, K.; et al. COVID-19 vaccine uptake among healthcare workers in the fourth country to authorize BNT162b2 during the first month of rollout. Vaccine 2021, 39, 5762-5768. [CrossRef]

27. Lwanga, S.K.; Lemeshow, S.; World Health Organization. Sample Size Determination in Health Studies: A Practical Manual; World Health Organization: Geneva, Switzerland, 1991. Available online: https://apps.who.int/iris/handle/10665/40062 (accessed on 15 October 2021). 
28. Israel, G.D. Determining Sample Size; Technical Report; University of Florida: Gainesville, FL, USA, 1992. Available online: https:/ / www.tarleton.edu/academicassessment/documents/samplesize.pdf (accessed on 5 January 2022).

29. Adam, M.; Gameraddin, M.; Alelyani, M.; Alshahrani, M.Y.; Gareeballah, A.; Ahmad, I.; Azzawi, A.; Komit, B.; Musa, A Evaluation of post-vaccination symptoms of two common COVID-19 vaccines used in Abha, Aseer Region, Kingdom of Saudi Arabia. Patient Prefer. Adherence 2021, 15, 1963-1970. [CrossRef] [PubMed]

30. Bendel, R.B.; Afifi, A.A. Comparison of stopping rules in forward "stepwise" regression. J. Am. Stat. Assoc. 1977, 72, 46-53.

31. Holm, S. A simple sequentially rejective multiple test procedure. Scand. J. Stat. 1979, 6, 65-70.

32. Gee, J.; Marquez, P.; Su, J.; Calvert, G.M.; Liu, R.; Myers, T.; Nair, N.; Martin, S.; Clark, T.; Markowitz, L.; et al. First month of COVID-19 vaccine safety monitoring-United States, December 14, 2020-January 13, 2021. Morb. Mortal. Wkly. Rep. 2021, 70, 283-288. [CrossRef]

33. Alhazmi, A.; Alamer, E.; Daws, D.; Hakami, M.; Darraj, M.; Abdelwahab, S.; Maghfuri, A.; Algaissi, A. Evaluation of side effects associated with COVID-19 vaccines in Saudi Arabia. Vaccines 2021, 9, 674. [CrossRef] [PubMed]

34. Al Bahrani, S.; Albarrak, A.; Alghamdi, O.A.; Alghamdi, M.A.; Hakami, F.H.; Al Abaadi, A.K.; Alkhrashi, S.A.; Alghamdi, M.Y.; Almershad, M.M.; Alenazi, M.M.; et al. Safety and reactogenicity of the ChAdOx1 (AZD1222) COVID-19 vaccine in Saudi Arabia. Int. J. Infect. Dis. 2021, 110, 359-362. [CrossRef]

35. Alghamdi, A.A.; Alkazemi, A.; Alissa, A.; Alghamdi, I.; Alwarafi, G.; Waggas, H.A. Adverse events following AstraZeneca COVID-19 vaccine in Saudi Arabia: A cross-sectional study among healthcare and non-healthcare workers. Intervirology 2021. [CrossRef]

36. Zaki, N.W.; Sidiq, M.; Qasim, M.; Aranas, B.; Hakamy, A.; Ruwais, N.; Alanezi, H.; Al Saudi, D.A.; Alshahrani, R.B.S.; Al-Thomali, A.B.A.; et al. Stress and psychological consequences of COVID-19 on health-care workers. J. Nat. Sci. Med. 2020, 3, $299-307$. [CrossRef]

37. Tissot, N.; Brunel, A.S.; Bozon, F.; Rosolen, B.; Chirouze, C.; Bouiller, K. Patients with history of COVID-19 had more side effects after the first dose of COVID-19 vaccine. Vaccine 2021, 39, 5087-5090. [CrossRef]

38. Alghamdi, A.N.; Alotaibi, M.I.; Alqahtani, A.S.; Al Aboud, D.; Abdel-Moneim, A.S. BNT162b2 and ChAdOx1 SARS-CoV-2 post-vaccination side-effects among Saudi vaccinees. Front. Med. 2021, 8, 760047. [CrossRef]

39. Abu-Hammad, O.; Alduraidi, H.; Abu-Hammad, S.; Alnazzawi, A.; Babkair, H.; Abu-Hammad, A.; Nourwali, I.; Qasem, F.; Dar-Odeh, N. Side effects reported by Jordanian healthcare workers who received COVID-19 vaccines. Vaccines $2021,9,577$. [CrossRef] [PubMed]

40. Andrzejczak-Grządko, S.; Czudy, Z.; Donderska, M. Side effects after COVID-19 vaccinations among residents of Poland. Eur. Rev. Med. Pharmacol. Sci. 2021, 25, 4418-4421. [CrossRef] [PubMed]

41. Jayadevan, R.; Shenoy, R.S.; Anithadevi, T.S. Survey of symptoms following COVID-19 vaccination in India. MedRxiv 2021 Available online: https://www.medrxiv.org/content/10.1101/2021.02.08.21251366v1.full.pdf (accessed on 20 November 2021).

42. Esba, L.; Al Jeraisy, M. Reported adverse effects following COVID-19 vaccination at a tertiary care hospital, focus on cerebral venous sinus thrombosis (CVST). Expert Rev. Vaccines 2021, 20, 1037-1042. [CrossRef] [PubMed]

43. Greinacher, A.; Thiele, T.; Warkentin, T.E.; Weisser, K.; Kyrle, P.A.; Eichinger, S. Thrombotic thrombocytopenia after ChAdOx1 nCov-19 vaccination. N. Engl. J. Med. 2021, 384, 2092-2101. [CrossRef]

44. Alfawaz, H.; Amer, O.E.; Aljumah, A.A.; Aldisi, D.A.; Enani, M.A.; Aljohani, N.J.; Alotaibi, N.H.; Alshingetti, N.; Alomar, S.Y.; Khattak, M.; et al. Effects of home quarantine during COVID-19 lockdown on physical activity and dietary habits of adults in Saudi Arabia. Sci. Rep. 2021, 11, 5904. [CrossRef] [PubMed]

45. Mohammed, R.A.; Garout, R.M.; Wahid, S.; Ayub, F.; Leen, M.; ZinAlddin, F.; Sultan, I. A survey on the side effects of Pfizer/BioNTech COVID-19 vaccine among vaccinated adults in Saudi Arabia. Cureus 2021, 13, e19222. [CrossRef]

46. Vassallo, A.; Shajahan, S.; Harris, K.; Hallam, L.; Hockham, C.; Womersley, K.; Woodward, M.; Sheel, M. Sex and gender in COVID-19 vaccine research: Substantial evidence gaps remain. Front. Glob. Women Health 2021, 2, 761511. [CrossRef]

47. Flanagan, K.L.; Fink, A.L.; Plebanski, M.; Klein, S.L. Sex and gender differences in the outcomes of vaccination over the life course Annu. Rev. Cell Dev. Biol. 2017, 33, 577-599. [CrossRef]

48. McCartney, P.R. Sex-based vaccine response in the context of COVID-19. J. Obstet. Gynecol. Neonatal Nurs. 2020, 49, 405-408. [CrossRef]

49. Harris, T.; Nair, J.; Fediurek, J.; Deeks, S.L. Assessment of sex-specific differences in adverse events following immunization reporting in Ontario, 2012-2015. Vaccine 2017, 35, 2600-2604. [CrossRef] [PubMed]

50. Riad, A.; Hocková, B.; Kantorová, L.; Slávik, R.; Spurná, L.; Stebel, A.; Havril'ak, M.; Klugar, M. Side effects of mRNA-based COVID-19 vaccine: Nationwide phase IV study among healthcare workers in Slovakia. Pharmaceuticals 2021, 14, 873. [CrossRef] [PubMed]

51. Jeon, M.; Kim, J.; Oh, C.E.; Lee, J.Y. Adverse events following immunization associated with the first and second doses of the ChAdOx1 nCoV-19 vaccine among healthcare workers in Korea. Vaccines 2021, 9, 1096. [CrossRef] [PubMed] 